\title{
MOEBIUS TRANSFORMATIONS AND CONTINUED FRACTIONS
}

\section{H. SCHWERDTFEGER}

R. E. Lane ${ }^{1}$ has shown how the criterion for convergence of a periodic continued fraction can be based upon simple properties of the linear fractional transformation (Moebius transformation)

$$
Z=A(z)=(a z+b) /(c z+d) .
$$

The method may be further adapted to the pure "transformation point of view" by: (a) interpretation of the two lemmas of Lane's paper as reductions of (1) to its similarity normal forms; (b) proving and expressing the final theorem in terms of the theory of the Moebius transformation only.

This will be done in the present note which can be read without reference to Lane's paper.

The Moebius transformation ${ }^{2}(1)$ is defined by its matrix

$$
A=\left(\begin{array}{ll}
a & b \\
c & d
\end{array}\right)
$$

of complex elements with nonvanishing determinant $\delta$, or by any matrix $\lambda A$ where $\lambda \neq 0$. Let $x_{1}, x_{2}$ be its fixed points: $A\left(x_{v}\right)=x_{v}$; then these are also the fixed points of the iterated transformations $z_{n}=A^{n}(z)(n=1,2, \cdots)$.

Let $T$ be the matrix of another Moebius transformation $z^{\prime}=T(z)$ by which the new variable $z^{\prime}$ is introduced. Then $T\left(x_{\nu}\right)$ are the fixed points of the transformation ${ }^{8}$

$$
Z^{\prime}=T A T^{-1}\left(z^{\prime}\right) .
$$

Suppose $x_{1} \neq x_{2}$; then $T\left(x_{1}\right) \neq T\left(x_{2}\right)$ and the transformation $T$ may be chosen so that $T\left(x_{1}\right)=0, T\left(x_{2}\right)=\infty$, namely, $z^{\prime}=\left(z-x_{1}\right) /\left(z-x_{2}\right)$, and (2) becomes

$$
Z^{\prime}=k z^{\prime}
$$

where $k=\left(a-c x_{1}\right) /\left(a-c x_{2}\right)$ is a complex constant, uniquely defined

Received by the editors September 18, 1945.

$1 \mathrm{R}$. E. Lane, The convergence and the values of periodic continued fractions, Bull. Amer. Math. Soc. vol. 51 (1945) pp. 246-250.

2 Cf. C. Caratheodory, Conformal representation, Cambridge, 1932, chap. 1, §26.

${ }^{3}$ The matrix of the product (that is, composition) of two Moebius transformations, first $A$, then $B$, is the product $B A$ of the two matrices. 
by $A$ and the same for all similar transformations $S A S^{-1}$. The transformation $A$ is said to be elliptic if $|k|=1(k \neq 1)$, hyperbolic if $k>0$, in all other cases loxodromic; it is the identity if and only if $k=1$.

If $x_{1}=x_{2}=x$, a transformation $T$ can be found so that $T(x)=\infty$; then $T A T^{-1}$ becomes a translation $Z^{\prime}=z^{\prime}+b^{\prime}$. By suitable choice of $T$ one can reach the normal form

$$
Z^{\prime}=z^{\prime}+1
$$

of $A$ which, in this case, is said to be parabolic.

For a given $z \neq x_{\nu}(\nu=1,2)$ we consider the recurring sequence $z_{n}=A\left(z_{n-1}\right)=\cdots=A^{n}(z)$. If it is convergent its limit is a fixed point $x_{1}$ of $A$. If $z_{n} \rightarrow x_{1}$ for all $z$ of a neighborhood of $x_{1}$, we call $x_{1}$ an attractive fixed point of $A$. At the same time $T\left(x_{1}\right)$ is an attractive fixed point of $T A T^{-1}$. If $x_{1} \neq x_{2}$ it follows from (3) that $x_{1}$ is attractive if and only if $|k|<1$, and then $z_{n} \rightarrow x_{1}$ for all $z \neq x_{2}$; therefore the fixed point $x_{2}$ is said to be repulsive.

Evidently $\infty$ is an attractive fixed point of the translation (4); thus the only fixed point $x$ of a parabolic transformation $A$ is always attractive. ${ }^{4}$

If $A$ is elliptic, $|k|=1$, the sequence $z_{n}$ is bounded and divergent whatever the initial point $z \neq x_{v}$; the points $z_{n}$ all lie on the circumference of the same invariant circle of $A$. The fixed points $x_{1}, x_{2}$ are symmetric with respect to this circle; they are called indifferent fixed points of $A$.

Now let

$$
f=\frac{a_{1}}{b_{1}}+\frac{a_{2}}{b_{2}}+\cdots
$$

be a given continued fraction where all $a_{\mu} \neq 0$. Its approximants $f_{q}$ may be represented in the following way: Let

$$
A_{1}=\left(\begin{array}{ll}
0 & a_{1} \\
1 & b_{1}
\end{array}\right), \quad A_{2}=\left(\begin{array}{ll}
0 & a_{2} \\
1 & b_{2}
\end{array}\right), \cdots
$$

Then one has

$$
\begin{gathered}
f_{0}=A_{1}(\infty)=0, \quad f_{1}=a_{1} / b_{1}=A_{1}(0)=A_{1} A_{2}(\infty), \cdots, \\
f_{q-1}=A_{1} A_{2} \cdots A_{q-1}(0)=A_{1} A_{2} \cdots A_{q}(\infty) .
\end{gathered}
$$

By definition let

4 In the book of G. Julia, Principes gêometriques d'analyse, I, Paris, 1930, it is stated on p. 30 that the fixed point of a parabolic transformation is indifferent. This depends, however, on the inadequate definition of "indifferent" given there on p. 23. 


$$
f_{-1}=\infty \text {. }
$$

Now let $f$ be periodic with a period of length $m$ so that for all $n=0,1,2, \cdots$

$$
a_{q}=a_{r}, \quad b_{q}=b_{r} \text { if } q=n m+r
$$

where $r$ may be the least non-negative remainder $(\bmod m)$ of $q$. We introduce the "period matrix"

$$
A=\lambda A_{1} A_{2} \cdots A_{m} \quad(\lambda \neq 0) .
$$

Then

$$
f_{q-1}=A^{n} A_{1} \cdots A_{r}(\infty)=A^{n}\left(f_{r-1}\right)
$$

for $r=0,1, \cdots, m-1$, whence the main result follows immediately:

The pernodic continued fraction $f$ is convergent if and only if the following three conditions are satisfied:

1. The Moebius transformation with the period matrix $A$ is not elliptic.

2. The attractive fixed point $x_{1}$ of $A$ is finite.

3. For all $r=0, \cdots, m-1$ one has $f_{r-1} \neq x_{2}$ where $x_{2}$ is the repulsive fixed point of $A$.

Then $f=x_{1}$.

Remark. For $r=0$ the condition 3 implies that also $x_{2}$ must be finite. There is no restriction for $f_{r-1}$ if $A$ is parabolic. The condition $1 \mathrm{im}$ plies Lane's inequality $(1,4)$. In fact $f_{m-1}=A(\infty)$ is the pole of the transformation $A^{-1}$ which has equal distance from both fixed points $x_{1}, x_{2}$ if and only if $A$ is elliptic. In fact from (3) one has $f_{m-1}-x_{1}$ $=k\left(f_{m-1}-x_{2}\right)$ whence moreover it follows at once that in the nonelliptic case the pole of $A^{-1}$ is nearer to the attractive fixed point of $A$.

For the application of the theorem it may be useful to have the necessary and sufficient condition for a Moebius transformation

$$
A=\left(\begin{array}{ll}
a & b \\
c & d
\end{array}\right)
$$

to be elliptic; it is

$$
\Re\left(\bar{\tau} \cdot\left(\tau^{2}-4 \delta\right)^{1 / 2}\right)=0
$$

where $\tau=a+d, \delta=a d-b c$, and $\tau$ the conjugate complex of $\tau$.

The University of Adelaide 